\title{
Photoelectric Method of Measuring Laser Divergence Angle
}

\author{
Xue Leng \\ School of Photoelectric Engineering, Changchun University of Science and Technology, Changchun, \\ 130000, China \\ lengxue79@126.com
}

\begin{abstract}
This article describes a test method for the pulsed laser beam divergence angle. Pulsed laser focused through the imaging optical system, the light spot formed on the CCD camera and image analysis by an image of the spot, the spot diameter is obtained, to calculate the value of the divergence angle of the laser beam. Experimental results show that this method can be more accurately measured slight angle high-energy pulsed laser beam divergence angle of value.
\end{abstract}

Keywords: Laser divergence angle; Laser spot; CCD.

\section{Introduction}

With the continuous development of laser technology, high-energy pulsed laser applications in the military is very extensive. Accurate measurement of beam quality it is essential for the assessment of the Operational Effectiveness of laser performance and evaluation of laser weapon systems. Laser beam divergence angle of the laser as an evaluation of the quality of an important parameter to accurately measure high-energy pulsed laser beam divergence angle is necessary.

\section{Measurement Method}

\subsection{Measuring principle}

In this paper, the use of photoelectric measurement of the laser beam divergence angle. Placed in the focal plane of the optical system aperture stop, an optical linear array or area array CCD acquisition aperture stop after the intensity distribution obtained after data processing beam divergence angle. This measurement method for measuring high precision test shown in Figure 1:

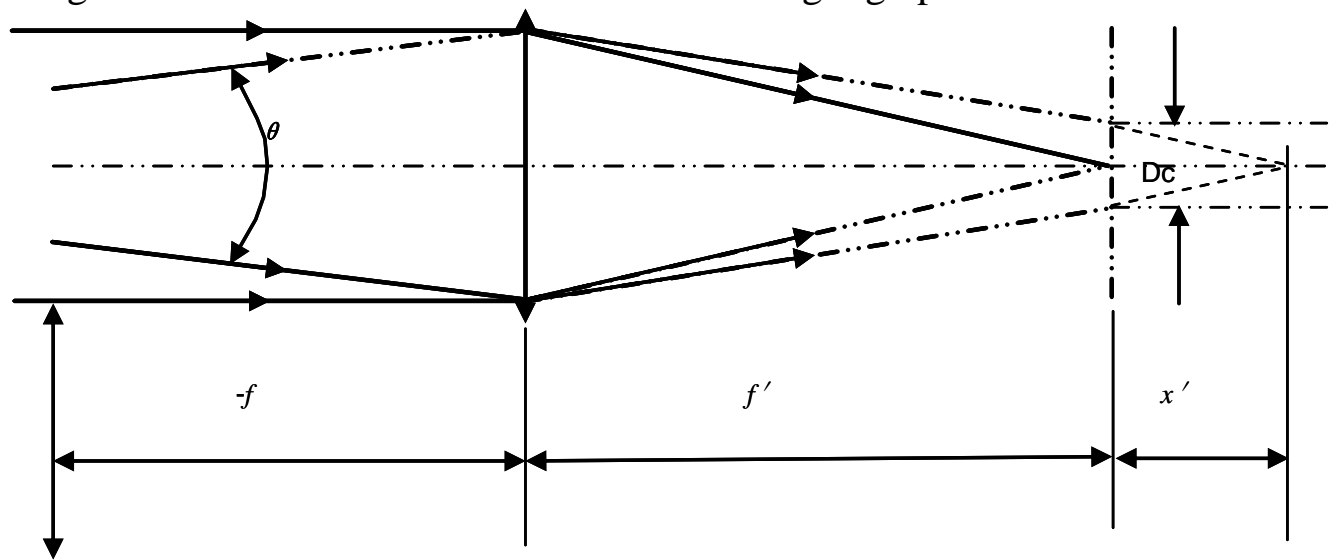

Fig1. Laser beam divergence angle measurement schematics

If the laser beam is a parallel light beam is incident on the beam divergence angle measurements of the optical system focused on the focal plane of the optical system if the aberration is small, in the focal plane to form a diffuse plaques, diffuse spot size depends on the beam divergence angle measurements of the optical system of the entrance pupil aperture size.

According to Newton's formula:

$-x x^{\prime}=f^{\prime 2}$

According to a similar triangle theorem: 
$\frac{D_{c}}{D}=\frac{x^{\prime}}{f^{\prime}+x^{\prime}}$

The laser divergence angle size is:

$\theta=2 \operatorname{arctg}\left(\frac{D}{2(-x-f)}\right)$

$\theta=2 \operatorname{arctg}\left(\frac{D_{c}}{2 f^{\prime}}\right)$

Since the measured divergence angle is small, so the equation simplifies to:

$\theta=\frac{D_{c}}{f^{\prime}}(\mathrm{Rad})$

If the exact value of the measured beam divergence angle tester optical system and the focal length of the laser spot diameter at its focal plane can calculate the divergence angle of the beam.

\subsection{Calculation spot center}

Laser beam spot center position is expressed as

$$
\begin{aligned}
& \overline{x_{c}}=\frac{\sum I(x, y) * x}{\sum I(x, y)} \\
& \overline{y_{c}}=\frac{\sum I(x, y)^{*} y}{\sum I(x, y)}
\end{aligned}
$$

\subsection{Laser spot size calculation}

In the Cartesian coordinate system, the $\mathrm{Z}$ beam at energy / power density function is expressed as the second moment.

$$
\begin{aligned}
\sigma_{x}^{2}(z) & =\frac{\sum I(x, y) *\left(x_{i}-\overline{x_{c}}\right)^{2}}{\sum I(x, y)} \\
\sigma_{y}{ }^{2}(z) & =\frac{\sum I(x, y) *\left(y_{i}-\overline{y_{c}}\right)^{2}}{\sum I(x, y)}
\end{aligned}
$$

Corresponding spot size is:

$$
\begin{aligned}
& \omega_{x}=2 \sigma_{x}(z) \\
& \omega_{y}=2 \sigma_{y}(z)
\end{aligned}
$$

As long as the measured spot size of the laser beam in the CCD image plane, you can obtain the laser beam divergence angle.

\subsection{Experimental Results}

Experimental selected wavelength $532 \mathrm{~nm}$ visible laser beam and a continuous wavelength of $1540 \mathrm{~nm}$ pulsed infrared laser beam is measured. Focal length $532 \mathrm{~nm}$ beam computed measurement of the focal length $1540 \mathrm{~nm}$ beam measurement is calculated. Laser spot experiments shown in Figure 2, 3.

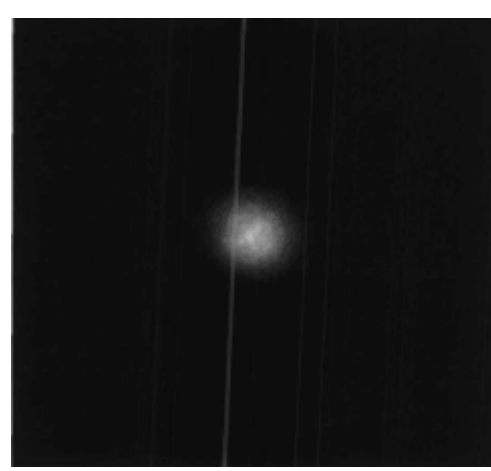

Fig2. 532nm beam spot pattern

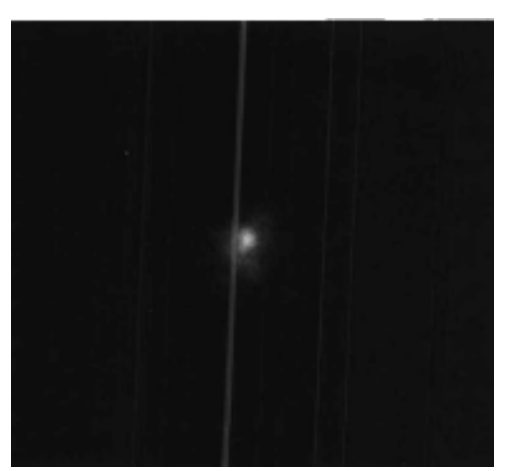

Fig3. 1540nm beam spot pattern 
Visible light CCD camera collected the resulting series of spots for data processing, the results are as follows.

Table1 532nm beam divergence angle of the beam measurements

\begin{tabular}{|c|c|c|c|c|c|c|c|c|}
\hline Numbers & 1 & 2 & 3 & 4 & 5 & 6 & 7 & 8 \\
\hline Beam & $\mathrm{X}: 0.95$ & X:0.96 & $\mathrm{X}: 0.97$ & X:0.96 & X:0.96 & X:0.96 & $\mathrm{X}: 1.00$ & $\mathrm{X}: 0.97$ \\
\hline angle(mrad) & Y:0.96 & Y:0.99 & $Y: 1.01$ & $Y: 1.00$ & Y:0.99 & $Y: 0.99$ & $Y: 1.03$ & Y:1.02 \\
\hline
\end{tabular}

The average of the data obtained.

$$
\begin{aligned}
& \bar{X}=0.966 \mathrm{mrad} \\
& \bar{Y}=0.999 \mathrm{mrad}
\end{aligned}
$$

Infrared CCD cameras capture the resulting series of spots for data processing, the results are as follows.

\begin{tabular}{|c|c|c|c|c|c|c|c|c|}
\hline Numbers & 1 & 2 & 3 & 4 & 5 & 6 & 7 & 8 \\
\hline Beam & $\mathrm{X}: 0.84$ & $\mathrm{X}: 0.86$ & $\mathrm{X}: 0.88$ & $\mathrm{X}: 0.85$ & $\mathrm{X}: 0.86$ & $\mathrm{X}: 0.82$ & $\mathrm{X}: 1.04$ & X:0.92 \\
\hline $\begin{array}{l}\text { divergence } \\
\text { angle(mrad) }\end{array}$ & $Y: 0.82$ & $Y: 0.85$ & Y:0.95 & $Y: 0.81$ & $Y: 0.94$ & Y:1.14 & $Y: 1.29$ & $Y: 0.89$ \\
\hline
\end{tabular}

Table2 1540 beam divergence angle of the beam measurements

The average of the data obtained.

$$
\begin{aligned}
& \bar{X}=0.878 \mathrm{mrad} \\
& \bar{Y}=0.948 \mathrm{mrad}
\end{aligned}
$$

\section{Summary}

Measurement methods used in this paper is simple, easy to implement, it is possible to achieve good accuracy. Able to complete the pulsed laser beam divergence angle measurements.

\section{References}

[1]. Lee Hong-Shik. Compace laser transmitter delivering a long-range infrared beam aligned with a monitoring visible beam. Applied optics.Vol51(2012) No17,P3936-

[2]. 3940

[3]. Hossain Md Arafat. Smartphone laser beam spatial profiler. Optics letters.Vol40

[4]. (2015)No22, P5156-5159.

[5]. Li Yanfang.The output power and beam divergence behaviors of tapered terahetz quantum cascade lasers. Optics express.Vol21 (2013) No13, P15998-16006.

[6]. Eckerskorn Nicko.Hollow Bessel-like beam as an optical guide for a stream of microsopiv particles. Optic express.Vol21 (2013) No25, p30492-30499. 